\title{
Digital Rock Evaluation of the Thermal Conductivity and Electrical Resistivity of Source Rocks
}

\author{
Shannon L. Eichmann ${ }^{1 *}$, Abdelrahman Kotb ${ }^{1}$, and Mita Sengupta ${ }^{1}$ \\ 1. Aramco Services Company: Aramco Research Center - Houston, Houston, TX USA \\ * Corresponding author: shannon.eichmann@aramcoservices.com
}

Unconventional reservoirs are chemically heterogeneous where the rock matrix is minerals and clays and the hydrocarbons are held in the pores of thermally matured organic matter all of which vary spatially at the nanoscale. This nanoscale heterogeneity poses challenges in measuring the petrophysical properties of source rocks and interpreting the data with reference to the changing rock structure. Focused ion beam scanning electron microscopy (FIB-SEM) is a common method used to study source rock structure where significant micro- and nanoscale heterogeneity exists.

Digital rock physics (DRP) provides an image-based means to characterize rock and petrophysical properties through image segmentation and advanced mathematical simulations. For rock characterization, DRP is used to complement slow or expensive laboratory measurements or to provide properties when accurate laboratory techniques do not exist [1-4]. A DRP approach allows the rock volume to be segmented into individual components such as matrix minerals, organic matter, pyrite, and pore space. After segmentation, these components are used to quantify changing mineralogy, organic matter content, as well as absolute permeability, and so allowing for quantitative analyses of FIB-SEM data. Recent advances in DRP have provided the ability to determine two-phase [2-4] and three-phase relative permeability [4], capillary pressure [3, 4], and electrical resistivity [5] where the two-phase and three-phase relative permeability predictions have been reported in literature for unconventional reservoir rocks $[2,3]$. In addition, a correlative simulation-based study of structural effects on mechanical, thermal and electrical conductivity, and flow properties has been reported for nanoporous metals and is directly relevant to unconventionals [6].

While permeability, absolute and relative, is an important property in reservoirs characterization because it affects overall hydrocarbon production it is not directly measured at the reservoir scale. In addition, measuring permeability accurately in the lab for ultra-low permeability rocks such as source rocks is challenging. Other petrophysical properties are also of interest such as thermal conductivity and electrical resistivity. Both of these properties can be measured on bulk samples both in the lab and in the borehole $[7,8]$ but few techniques exist that can measure these properties at the micro- and nanoscale, which is the length scale of the source rock heterogeneity. Bulk measurement of the thermal conductivity and electrical resistivity does not make it possible to study their dependence on the local rock structure for highly heterogeneous rocks such as source rocks. While permeability prediction using a DRP approach has been demonstrated for unconventionals, a DRP study of the thermal conductivity and electrical resistivity is less commonly reported in the literature.

Here we demonstrate a DRP study of how source rock composition, structure, and porosity affect thermal conductivity and electrical resistivity using FIB-SEM images of source rock samples. Figure 1a and $1 \mathrm{~b}$ show single representative secondary electron (SE) and backscatter electron (BSE) slices. In this work, the individual SE and BSE slices are aligned, cropped, and combined to create a three-dimensional image (Figure 1c), which is then segmented (Figure 1d) prior to numerical simulations. Full cubes such as those in Figure 1c were additionally sub-sectioned into eight sub-cubes and segmented to determine the amount 
of the matrix minerals (i.e., calcite), organic matter, pyrite, and pore space. The segmented full cubes and sub-cubes were used to determine the thermal conductivity, electrical resistivity, and permeability in the $\mathrm{X}, \mathrm{Y}$, and $\mathrm{Z}$ directions using the GeoDict ConductoDict and FlowDict modules.

The data show that the DRP approach predicts a decrease in thermal conductivity with increasing porosity $(\phi)$ for $\phi>0.05$. When the porosity was lower than 5 percent, the effects of the changing matrix composition are dominant. The DRP-predicted electrical resistivity predictions show an interesting trend with absolute permeability when a fully water saturated state is assigned to the pore space and additional studies will be done to explore the effects of water saturation. The thermal conductivity and electrical resistivity results are compared to structural variations and the anisotropy of the thermal and electrical properties is also included. DRP predicted thermal conductivity and electrical resistivity provide a possible method to link nanoscale rock properties to properties that can be physically measured at the lab and reservoir scales and may lead to future improvements in log interpretation [9].

[1] N. Saxena et al., Advances in Water Resources 109 (2017), p. 211.

[2] A.P. Byrnes et al., Unconventional Resources Technology Conference (2017) p. 1584.

[3] A.P. Byrnes et al., Unconventional Resources Technology Conference (2018) p. 3111.

[4] S. Berg et al., Advances in Water Resources 90 (2016), p. 24.

[5] M. Halisch et al., Proceedings, Annual Meeting of the Society of Core Analysts (2015) p.1.

[6] X. Zheng et al., The Journal of Physical Chemistry C 122 (2018), p. 16803.

[7] T.J. Ahrens "Rock Physics \& Phase Relations" (American Geophysical Union).

[8] J.H. Schön "Physical properties of rocks: Fundamentals and principles of petrophysics" (Elsevier).

[9] We would like to thank Jordan Kone and Josh Brothers for FIB-SEM data collection.
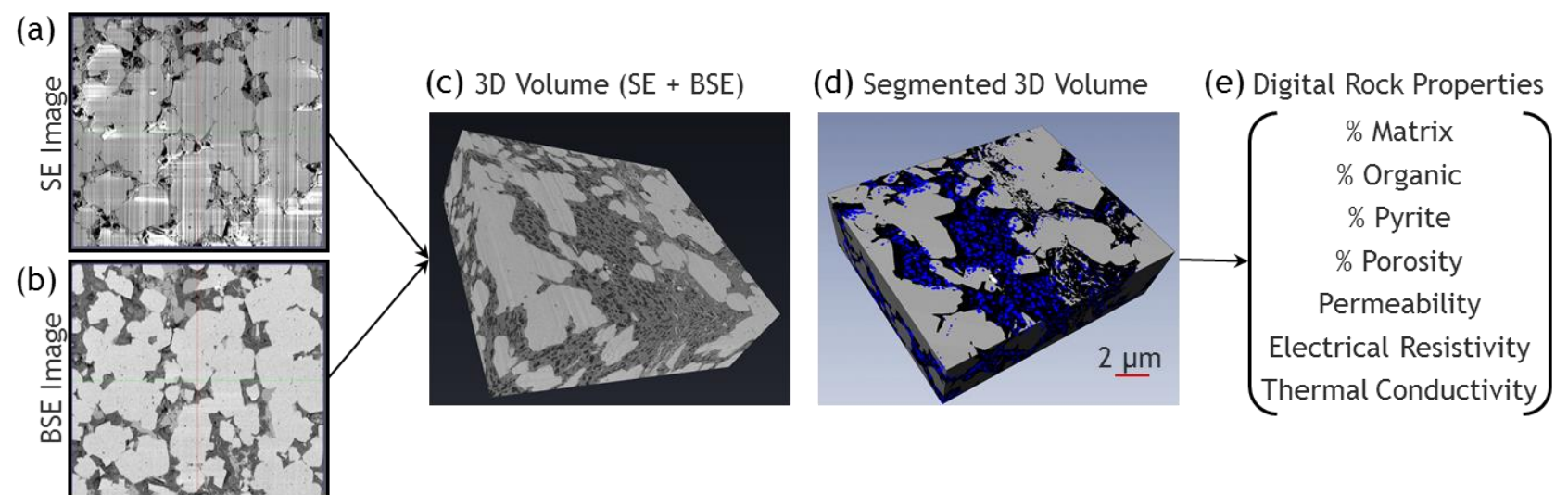

Figure 1: Representative ( $a, b)$ secondary electron (SE) and backscatter electron (BSE) images from a single FIB-SEM slice, (c) combined SE and BSE three dimensional FIB-SEM volume, (d) segmented sample volume used for DRP analyses, and (e) DRP measured properties for data analyses. 